MODELING, IDENTIFICATION AND CONTROL, 2000, vOL. 21, NO. 2, 65-82

doi:10.4173/mic.2000.2.1

\title{
Identification of Vibration Parameters in a Spacecraft using Kalman Filters, Numerical Search and Phase-Locked Loops
}

\author{
AAGE SKULLESTAD† \\ Keywords: Space structures, parameter identification, Kalman filters, phase-locked \\ loops \\ Communication in space requires antennas that are accurately pointed. Often \\ antennas are mounted on lightly damped space structures, that are easily set into \\ vibration, which decreases the pointing accuracy of the antennas. Knowing the \\ vibration frequencies simplifies the control tasks and may also improve the pointing \\ accuracy. This paper describes identification of vibration frequencies in a space \\ structure, based on frequency weighted Kalman filters, a Gauss-Newton numerical \\ search method and phase-locked loops.
}

\section{Introduction}

Future utilization of space is expected to require large space structures. Because of their light weight, these structures will tend to have low damping and vibration frequencies (natural frequencies) that will be closely spaced and are expected to vary as much as $20-30 \%$, due to splashing and consumption of rocket fuel. Some of the vibration frequencies are expected to be lower than the bandwidths of the control loops. An increasing need for better pointing accuracy of antennas connected to the space structure puts strong demands on the control system. Thus, knowing the vibration frequencies will be of vital interest and the need for identifying the vibration frequencies in space is inherent.

This paper presents identification of vibration parameters using an augmented Kalman filter, a frequency weighted Kalman filter, and a prediction-error-method (PEM) using a Gauss-Newton numerical search to perform the minimization and phase-locked loops (PLL). A flexible Euler-Bernoulli beam connected to a rigid satellite core is modelled as the space structure. The flexible beam is forced to vibrate by applying an initial displacement of the beam. A sensor located at the end of the beam then measures the displacement of the beam.

AR, ARX, ARMA and ARMAX models are quite common in parameter identification (Söderström et al., 1989 and Ljung, 1987). However, parameter identification may also be performed using state-space models. These models are often easier to understand than the difference equations employed in AR, ARX, ARMA and ARMAX models. Augmented Kalman filters (Gelb, 1974), which are based on statespace models, are known to perform parameter identification as well as state estimation. Frequency weighted augmented Kalman filters may be used to improve the parameter accuracy compared to ordinary augmented Kalman filters (Williamson, 1991). Another method that is reported to give accurate parameter identification (Ljung, 1987), is the Gauss-Newton numerical search applied to a PEM. Phase-locked loops are normally used at the receiver end to generate a local carrier that is locked in

$\dagger$ Kongsberg Defence \& Aerospace, Pb. 1003, 3600 Kongsberg. 
phase and frequency to that of the receiver signal (Proakis et al., 1988). PLLs are also shown to be applicable to vibration frequency estimation (ESA Contract No. 4834/ 81,1990 ).

Section 2 presents the mathematical model of the spacecraft. Section 3 describes parameter identification using an augmented Kalman filter, a frequency weighted Kalman filter, a Gauss-Newton numerical search and PLLs. The properties of these methods are demonstrated by simulations in Section 4 . Section 5 concludes the paper.

\section{Mathematical model of a space structure}

The space structure consists of a flexible beam connected to a satellite body, Figure 1. The flexible beam is modelled as an Euler-Bernoulli beam and the satellite body as a rigid body. The reason for this is that the satellite body has a very stiff construction, whereas the beam has a light construction and is highly flexible. The beam is free to move in the y-direction only. The displacement-time function of an Euler-Bernoulli beam undergoing vibrations is described by the differential equation

$$
E J \frac{\partial^{4} y(x, t)}{\partial x^{4}}+\rho \frac{\partial^{2} y(x, t)}{\partial t^{2}}=f(x, t)
$$

where $\rho$ is the mass/unit length, $E$ is Young's modulus, $J$ is the moment of inertia and $f(x, t)$ is an external force per unit length of the beam. (1) has the solution (Rao, 1990)

$$
y(x, t)=\sum_{n=1}^{N} W_{n}(x) q_{n}(t)
$$

The displacement of the beam $y(x, t)$ is a function of time and the distance $x$ from the fixed beam end (connection satellite body/flexible beam): $N$ is the total number of vibration modes, $q_{n}(t)$ is the time dependent vibration of mode $n$ and $W_{n}(x)$ is the mode shape function of the $n^{\text {th }}$ vibration. $W_{n}(x)$ may be found from an ordinary textbook in mechanical engineering, for instance Rao (1990).

A flexible beam has an infinite number of vibration modes, however, most of them have low energy and contribute little to the displacement of the beam. The number of vibrations is in this paper limited to 4 . Table 1 shows the vibration frequencies of the flexible beam.

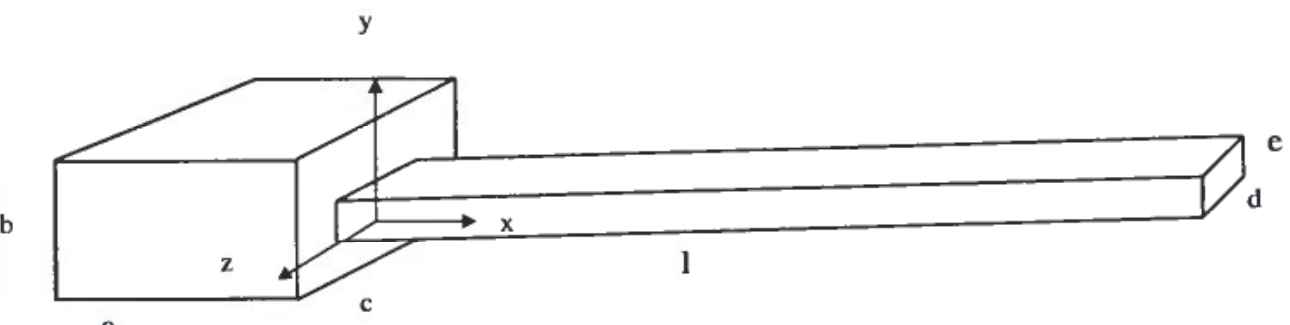

Figure 1. Spacecraft model.

Table 1. Frequencies of the beam vibrations

\begin{tabular}{lcccc}
\hline & \multicolumn{4}{c}{ Frequencies of the beam vibrations } \\
\hline Modes & 1 & 2 & 3 & 4 \\
Frequencies (Hz) & 0.51 & 3.22 & 3.50 & 9.02 \\
\hline
\end{tabular}


The vibration frequencies of the chosen beam are relatively widely spaced and mode 3 is modified in shape and frequency such that the frequencies of modes 2 and 3 become very narrowly spaced, as shown in Table 1 . The flexible beam is fixed to the satellite body, which is considered to be rigid. Also assuming the flexible beam to be rigid, the equation of motion referred to a body fixed frame of reference (principal axes $x, y, z$ in Figure 1) may be written (Skullestad, 1995)

$$
\begin{aligned}
& \left(J_{B}+J_{E}\right) \dot{\omega}+m_{B} \tilde{r}_{0} a_{0}+m_{E} \tilde{r}_{E} a_{0}=T_{B}+T_{E} \\
& \left(m_{B}+m_{E}\right) a_{0}-m_{B} \tilde{r}_{0} \dot{\omega}-m_{E} \hat{r}_{E} \dot{\omega}=F_{B}+F_{E}
\end{aligned}
$$

where $J_{B}$ and $J_{E}$ are the inertia of the satellite body and the beam, respectively, $\dot{\omega}$ is the angular velocity of frame $\mathrm{x}, \mathrm{y}, \mathrm{z}$ in Figure 1 referred to inertial space, $m_{B}$ and $m_{E}$ are the mass of the satellite body and the beam, respectively, $a_{0}$ is the acceleration of frame $\mathrm{x}, \mathrm{y}, \mathrm{z}$ referred to inertial space. $T_{B}$ and $T_{E}$ are the external moments acting on the satellite body and the beam respectively. $F_{B}$ and $F_{E}$ are the external forces acting on the satellite body and the beam respectively, $\tilde{r}$ is the skew symmetric matrix representation of a vector $r$ cross product.

(3) assumes the flexible beam to be rigid, that is not so and the vibrations of the flexible beam modify both the moment and the force equation in (3) to (De Lafountaine, 1990)

$$
\begin{aligned}
& \left(J_{B}+J_{E}\right) \dot{\omega}+m_{B} \tilde{r}_{0} a_{0}+m_{E} \tilde{r}_{E} a_{0}+\sum_{n=1}^{4} h_{n}(r) \ddot{q}_{n}(t)=T_{B}+T_{E} \\
& \left(m_{B}+m_{E}\right) a_{0}-m_{B} \tilde{r}_{0} \dot{\omega}-m_{E} \tilde{r}_{E} \dot{\omega}+\sum_{n=1}^{4} p_{n}(r) \ddot{q}_{n}(t)=F_{B}+F_{E}
\end{aligned}
$$

where $p_{n}$ is called the momentum coefficient and indicate to what extent mode $n$ participates in the translation of the body, $h_{n}$ is called the angular moment coefficient and indicate how much mode $n$ participate in the rotation of the body. (4) may be put into a general equation of motion. (5).

$$
M \ddot{x}+D \dot{x}+K x=F
$$

where $M$ is the mass matrix, $D$ is the damping matrix and $K$ is the stiffness matrix. The state vector $x$ is defined as:

$$
x=\left[\begin{array}{llllllllllllllllllll}
\theta_{x} & \theta_{y} & \theta_{z} & x & y & z & q_{1} & q_{2} & q_{3} & q_{4} & \dot{\theta}_{x} & \dot{\theta}_{y} & \dot{\theta}_{z} & \dot{x} & \dot{y} & \dot{z} & \dot{q}_{1} & \dot{q}_{2} & \dot{q}_{3} & \dot{q}_{4}
\end{array}\right]^{T}
$$

(5) may be converted into a state space model, (6)

$$
\begin{aligned}
& \dot{x}=A x+B u \\
& y=C x+D u
\end{aligned}
$$

where

$$
A=\left[\begin{array}{cc}
0 & I \\
-M^{-1} K & -M^{-1} D
\end{array}\right], \quad B=\left[\begin{array}{c}
0 \\
M^{-1}
\end{array}\right]
$$




\section{Geometrical Parameters}

The geometrical parameters are chosen to be:

$\begin{array}{ll}\text { Satellite body: } & \text { Mass: } 270 \mathrm{~kg} \\ & \text { Width }=\text { length }=\text { height: } 10 \mathrm{~m} \\ \text { Flexible beam: } & \text { Length: } 4 \mathrm{~m} \\ & \text { Width: } 0.05 \mathrm{~m} \\ & \text { Height: } 0.01 \mathrm{~m} \\ & \text { Mass density }=2700 \mathrm{~kg} / \mathrm{m}^{3} \\ & \text { Elasticity }=70 \mathrm{E}+9 \mathrm{~N} / \mathrm{m}^{2}\end{array}$

\section{Identification model to be used for the Kalman filters}

Parameter identification may be performed adding a mathematical model for each of the required parameters. In our case the complete model contains 20 states + four parameter models and, specially, if the number of vibrations is increased, this model becomes very complex. It is possible to simplify the above mathematical model. Looking at Figure 1, the flexible beam is, due to the geometrical parameters, free to move in the $\mathrm{y}$-direction only. A torque about the $\mathrm{x}$-axis or $\mathrm{y}$-axis or a force in the $x$ or $z$ direction will only to a small extent excite the vibrations. Therefore, it may be possible to remove those states from the identification model without losing accuracy. The time function of the vibrations may be found from the general equation of a simple harmonic motion, (7).

$$
\ddot{q}_{n}+2 \xi_{n} \omega_{n} \dot{q}_{n}+\omega_{n}^{2} q_{n}=f_{d}
$$

where $n$ is the mode number, $\xi_{n}$ is the damping coefficient of mode $n, \omega_{n}$ is the natural frequency of mode $n$ and $f_{d}$ is an external force.

Equation (7) written in component form using four vibrations (modes) becomes

$$
\left[\begin{array}{c}
\dot{x}_{1} \\
\dot{x}_{2} \\
\dot{x}_{3} \\
\dot{x}_{4} \\
\dot{x}_{5} \\
\dot{x}_{6} \\
\dot{x}_{7} \\
\dot{x}_{8} \\
\dot{x}_{9} \\
\dot{x}_{10} \\
\dot{x}_{11} \\
\dot{x}_{12}
\end{array}\right]=\left[\begin{array}{cccccccccccc}
0 & 0 & 0 & 0 & 1 & 0 & 0 & 0 & 0 & 0 & 0 & 0 \\
0 & 0 & 0 & 0 & 0 & 1 & 0 & 0 & 0 & 0 & 0 & 0 \\
0 & 0 & 0 & 0 & 0 & 0 & 1 & 0 & 0 & 0 & 0 & 0 \\
0 & 0 & 0 & 0 & 0 & 0 & 0 & 1 & 0 & 0 & 0 & 0 \\
\omega_{1}^{2} & 0 & 0 & 0 & -2 \xi \omega_{1} & 0 & 0 & 0 & 0 & 0 & 0 & 0 \\
0 & \omega_{2}^{2} & 0 & 0 & 0 & -2 \xi \omega_{2} & 0 & 0 & 0 & 0 & 0 & 0 \\
0 & 0 & \omega_{3}^{2} & 0 & 0 & 0 & -2 \xi \omega_{3} & 0 & 0 & 0 & 0 & 0 \\
0 & 0 & 0 & \omega_{4}^{2} & 0 & 0 & 0 & -2 \xi \omega_{4} & 0 & 0 & 0 & 0 \\
0 & 0 & 0 & 0 & 0 & 0 & 0 & 0 & 0 & 0 & 0 & 0 \\
0 & 0 & 0 & 0 & 0 & 0 & 0 & 0 & 0 & 0 & 0 & 0 \\
0 & 0 & 0 & 0 & 0 & 0 & 0 & 0 & 0 & 0 & 0 & 0 \\
0 & 0 & 0 & 0 & 0 & 0 & 0 & 0 & 0 & 0 & 0 & 0
\end{array}\right]\left[\begin{array}{l}
x_{1} \\
x_{2} \\
x_{3} \\
x_{4} \\
x_{5} \\
x_{6} \\
x_{7} \\
x_{8} \\
x_{9} \\
x_{10} \\
x_{11} \\
x_{12}
\end{array}\right]+\left[\begin{array}{l}
0 \\
0 \\
0 \\
0 \\
1 \\
1 \\
1 \\
1 \\
\frac{F}{m}
\end{array}\right]\left[\begin{array}{l}
w_{1} \\
w_{2} \\
w_{3} \\
w_{4} \\
w_{5} \\
w_{6} \\
w_{7} \\
w_{8} \\
w_{9} \\
w_{10} \\
w_{11} \\
w_{12}
\end{array}\right]
$$

where $m$ is the mass, $F$ is the force, $x_{1}-x_{8}$ represent the mathematical model of the beam, $x_{9}-x_{12}$ represent the parameter model of the vibration frequencies, and $w_{1}-w_{12}$ are the process noise. 
(8) may be used as the identification model without loosing significant accuracy. The sensor measures the displacement in the y-direction, i.e. perpendicular to the beam. In the case of position measurements, the measurement model becomes

$$
C=\left[\begin{array}{llllllll}
W_{1}(x) & W_{2}(x) & W_{3}(x) & W_{4}(x) & 0 & 0 & 0 & 0
\end{array}\right]
$$

The acceleration may be modelled as

$$
C=\left[\begin{array}{llll}
-\omega_{1}^{2} W_{1}(x) & -\omega_{2}^{2} W_{2}(x) & -\omega_{3}^{2} W_{3}(x) & -\omega_{4}^{2} W_{4}(x) \\
-2 \xi \omega_{1} W_{1}(x) & -2 \xi \omega W_{2}(x) & -2 \xi \omega W_{3}(x) & -2 \xi \omega_{4} W_{4}(x)
\end{array}\right]
$$

Which of these measurement matrices that are going to be used, depends on the chosen type of sensor. An accelerometer is an interesting sensor because of its large frequency range and is used in this paper. The accelerometer is located at the end of the beam and equations (8) and (10) represent the identification model. The true measurements are generated from the complete mathematical model (6) containing 20 states.

\section{Methods}

\subsection{Kalman filters}

An ordinary Kalman filter performs an optimal estimation of states $x$ in a linear stochastic system with known structure and parameters. Optimal in the sense that the estimated states have minimum variance. A linear discrete Kalman filter may be written (Gelb, 1974)

$$
\begin{gathered}
x(k+1)=\phi x(k)+\Delta u(k)+\Gamma w(k) \\
y(k)=C x(k)+v(k) \\
\bar{x}(k+1)=\phi \hat{x}(k)+\Delta u(k)+\Gamma \bar{w}(k) \\
\bar{P}(k+1)=\phi \hat{P}(k) \phi^{T}+\Gamma Q_{d} \Gamma^{T} \\
\hat{x}(k)=\bar{x}(k)+K(k)(y(k)-C \bar{x}(k)) \\
\hat{P}(k)=(I-K(k) C) \bar{P}(k) \\
K(k)=\bar{P}(k) C^{T}\left(C \bar{P}(k) C^{T}+R_{d}\right)^{-1}
\end{gathered}
$$

In the above equations:

$x(k)$ is the state vector

$\bar{x}(k)$ is the predicted states or the a priori states

$\hat{x}(k)$ is the estimated states or the a posteriori states

$w(k)$ is the process noise

$v(k)$ is the measurement noise

$\bar{P}(k)$ is the a priori error covariance matrix

$\hat{P}(k)$ is the a posteriori error covariance matrix

$Q_{d}$ is the process noise covariance matrix

$R_{d}$ is the measurement noise covariance matrix 
The process noise covariance matrix- $Q_{d}$

The process is often continuous-time, and so is $Q$. In the case of a discrete-time Kalman filter, (14) needs the expression $\Gamma Q_{d} \Gamma^{T}$, this expression may be found, given the spectral density (PSD) matrix $Q$, from

$$
\Gamma Q_{d} \Gamma^{T}=\int_{k}^{k+1} \phi\left(t_{k+1}, \tau\right) G Q G^{T}\left(t_{k+1}, \tau\right) d \tau
$$

where

$\Gamma$ is the discrete-time process noise weighting matrix

$G$ is the continuous-time process noise weighting matrix

$\phi$ is the transition matrix

If the time $T$ is small we may use a first order approximation, $\phi=I+\mathrm{A} T$, and (18) becomes

$$
\Gamma Q_{d} \Gamma^{T}=G Q G^{T} T
$$

\section{Measurement noise covariance matrix $-\boldsymbol{R}_{d}$}

If the measurements are continuous, the PSD matrix may be transformed to a discrete covariance matrix by

$$
R_{\mathrm{d}}=R / T
$$

\section{Parameter and state estimation-augmented Kalman filter}

The problem of identifying parameters in a system may be regarded as a special case of the general state estimation problem. If both the parameter vector $\theta$ and the state vector $x$ are to be estimated, we can combine them into a composite state vector $x^{\prime}(k)$.

$$
x^{\prime}(k)=\left[\begin{array}{c}
x_{1}(k+1) \\
0(k+1)
\end{array}\right]=\left[\begin{array}{c}
f\left(x_{1}(k), \theta(k), u(k), w_{1}(k)\right) \\
\theta(k)+w_{2}(k)
\end{array}\right]
$$

where

$x_{1}(k)$ is the process vector

$\theta(k)=\left[0_{1} \theta_{2} \ldots \theta_{n}\right]$ is the parameter vector

$w_{1}(k)$ and $w_{2}(k)$ are the process and parameter noise, respectively.

The Kalman gain, the process and measurement covariance matrices also consist of two parts, one for the states and one for the parameters. The covariance matrices are given as

$$
Q_{d}(k)=\left[\begin{array}{cc}
Q_{1}(k) & 0 \\
0 & Q_{2}(k)
\end{array}\right], \quad R_{d}(k)=\left[\begin{array}{cc}
R_{1}(k) & 0 \\
0 & R_{2}(k)
\end{array}\right]
$$

The a priori and a posteriori covariance matrices are defined as

$$
\bar{P}(k)=\left[\begin{array}{ll}
\bar{P}_{11}(k) & \bar{P}_{12}(k) \\
\bar{P}_{21}(k) & \bar{P}_{22}(k)
\end{array}\right], \quad \hat{P}(k)=\left[\begin{array}{ll}
\hat{P}_{11}(k) & \hat{P}_{12}(k) \\
\hat{P}_{21}(k) & \hat{P}_{22}(k)
\end{array}\right]
$$


Even if the original process is linear, the augmented model generally becomes nonlinear. The following linearization has to be made

$$
\phi(k)=\frac{\partial f(\cdot)}{\partial x(k)}\left|\begin{array}{l}
x(k)=\hat{x}(k) \\
u(k)=u(k) \\
w(k)=w(k),
\end{array} \quad C(k)=\frac{\partial c(\cdot)}{\partial x(k)}\right| x(k)=\hat{x}(k)
$$

\subsection{Frequency weighting/shaping}

The measurements used in a Kalman filter often consist of a mixture of strong and weak signals including measurement noise, and it may be difficult to estimate all states and/or parameters with expected accuracy. This is also a situation that often occurs in identification of vibrations. The energy of the different vibration modes, which depends on the physical system and the excitation of the physical system, will always differ. The frequencies of the low energy vibrations are normally difficult to identify.

Anderson et al. (1989) describe frequency weighting in connection with linear quadratic feedback. It may also be possible to apply frequency weighting in connection with state/parameter estimating. Maybeck (1979) and Williamson (1991) describe a frequency weighted Kalman filter. Suppose a process is described by

$$
\begin{aligned}
x(k+1) & =\phi x(k)+\Delta u(k)+\Gamma u(k) \\
y(k) & =C x(k)+v(k)
\end{aligned}
$$

The states and/or parameters of (25) can be estimated using an augmented Kalman filter. The measurements can be filtered by a user specified frequency weighting filter before being applied to the augmented Kalman filter. The discrete frequency weighted filter may be described as

$$
\begin{aligned}
x_{f}(k+1) & =\phi_{f} x_{f}(k)+\Delta_{f} v(k) \\
y_{f}(k) & =C_{f} x_{f}(k)
\end{aligned}
$$

The frequency weighted Kalman filter consists of (25) and (26) with states $x_{t}(k)=\left[x(k)^{T} x_{f}(k)^{T}\right]^{T}$ and is described by

$$
\begin{aligned}
x_{t}(k+1) & =\phi_{t} x_{t}(k)+\Delta_{t} u(k)+w_{t}(k) \\
y_{t}(k) & =C_{t} x_{t}(k)
\end{aligned}
$$

where

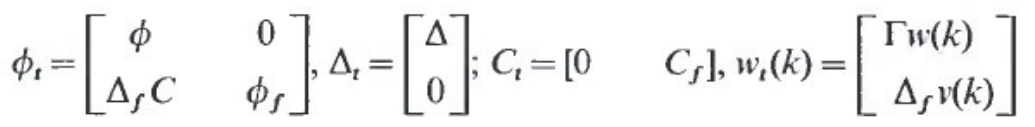

The augmented covariance matrix (14) modifies to

$$
\bar{P}(k+1)=\phi_{t} \hat{P}(k) \phi_{t}^{T}+\left[\begin{array}{cc}
\Gamma Q_{d} \Gamma^{T} & 0 \\
0 & \Delta_{f} R_{d} \Delta_{f}^{T}
\end{array}\right]
$$

In the above we have assumed $w(k) \sim N\left(0, Q_{d}\right)$ to be the process noise of the physical system whereas $v(k) \sim N\left(0, R_{d}\right)$ is the measurement noise of the system. 
The Kalman gain matrix modifies to:

$$
K(k)=\bar{P}(k) C_{f}^{T}\left(C_{f} \bar{P}(k) C_{f}^{T}+R_{f}\right)^{-1}
$$

Ideally, $R_{f}=0$, since the output comes from a frequency weighted filter, but experience shows that $R_{f}$ may be used in tuning the filter.

\subsection{Gauss-Newton numerical search}

In sections 3.1 and 3.2 an augmented Kalman filter and a frequency weighted Kalman filter were used to identify the vibration frequencies of the spacecraft. It is known (Skullestad, 1995) that numerical search methods perform better in the case of noisy measurements. Different numerical search methods are available, the GaussNewton iterative minimization routine is effective, especially close to minimum (Ljung, 1987) and is chosen here. The Gauss-Newton numerical search method may be expressed as

$$
\hat{\theta}_{N}^{(i+1)}=\hat{\theta}_{N}^{(i)}-\mu_{N}^{(i)}\left[R_{N}^{(i)}\right]^{-1} L_{N}^{\prime}\left(\hat{\theta}_{N}^{(i)}\right)
$$

where

$\hat{\theta}_{N}^{(i)}$ denotes the $i^{\text {th }}$ iteration

$L_{N}^{\prime}\left(\hat{\theta}_{N}^{(i)}\right)$ is the gradient of the loss function $L_{N}(\hat{\theta})$

$R_{N}^{(i)}$ modifies the search direction

$\mu_{N}^{(i)}$ is the step size

$R_{N}$ and $L_{N}^{\prime}(\theta)$ together determine the search direction and $\mu_{N}$ is normally taken as 1 and then divided by 2 for each new search, until the loss function has gained its required minimum value. A benefit of the numerical search method is the ability to use a priori information. If the values of the parameters to be estimated are totally unknown, a suboptimal analytical method usually is used to generate a set of initial conditions. If we, however, from earlier experiments or from physical knowledge of the process know more about the unknown parameters than an initial guess with a suboptimal method can give, these values should be given as a priori information. The computation may go much faster if the numerical method can start with accurate a priori values and the parameter estimates may also become more accurate.

A problem with numerical search methods is local minima, as one never knows for sure whether the parameter estimates are local minima, or global minima. Accurate a priori information may reduce the problem of taking local minima as true parameter values, but it is in any case recommended to make several identification runs, using different parameter initializations.

Ljung, 1987 describes Gauss-Newton numerical search minimizing the prediction error in both equation error models and state space models. The Kalman filter structure, (31), seemed to give the best results, applied to our problem, and identification using this model is documented in Section 4.3.

$$
\begin{aligned}
x(k+1) & =\phi x(k)+\Delta u(k)+K e(k) \\
y(k) & =C x(k)+e(k)
\end{aligned}
$$

The PEM function requires the model to be a Kalman filter structure of innovation type. The Kalman gain may be calculated as the steady state gain. Identification can be accomplished using the function PEM in MATLABs System Identification Toolbox.

The above method is also known as a prediction-error-method (PEM), since the 
method makes use of the prediction error. Sometimes the Gauss-Newton method is called a maximum likelihood (ML) method since the Gauss-Newton algorithm give results equal to ML results, in the case of Gaussian distributed disturbances, which is the case here.

\subsection{Phase locked loop}

PLLs may be used to detect vibration frequencies in a flexible structure. A PLL is shown in Figure 2.

A PLL may be used to generate a local sinusoidal that is locked in phase and frequency to that of the input signal (Proakis et al., 1988). The accelerometer output is used as input to the input-filter. The phase angle of the voltage controlled oscillator, $\mathrm{VCO}$, output is compared to the phase angle of the input signal, and the phase difference is used to modulate the VCO frequency. The VCO should ideally generate a sinusoidal with frequency equal to one of the vibration frequencies of the input signal. The low-pass filter (LP) removes unwanted frequencies that are generated from the phase detector. The controller is applied to select which frequency region that is to be tracked by the PLL, and to attenuate interfering signals. Sinusoidals (vibrations) with closely spaced frequencies (interfering signals) together with measurement noise degenerate the accuracy of the PLL. An input-filter, e.g. a band-pass filter connected to the input of the PLL may be applied in order to improve the frequency accuracy.

The phase detector multiply the input signal and the VCO output signal. The input signal may be written in the time domain as (ESA Contract No. 4834/81, 1990)

$$
u(t)=\sum_{i=1}^{N} A_{i} \sin \left(\omega_{i} t+\varphi_{i}\right)
$$

and the output signal from the VCO becomes

$$
y(t)=B \cos \left(\omega_{0} t+\hat{\varphi}(t)\right)
$$

where

$A_{i}$ - the amplitude of mode $i$

$\omega_{i}$ - the frequency of mode $i$

$\varphi_{i}$ - the phase lag of mode $i$

$B$ - the output amplitude of the VCO

$\omega_{0}$ - the output frequency of the VCO

$\hat{\varphi}(t)$ - the estimated phase of the VCO

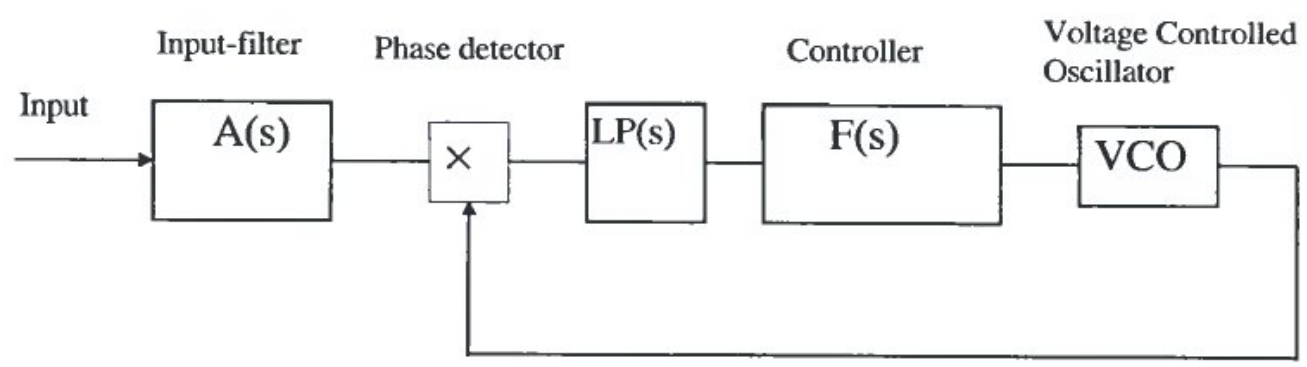

Figure 2. Phase-locked loop. 
The output from the phase detector becomes

$$
u(t) y(t)=\sum_{i=1}^{N} \frac{A_{i} B}{2}\left[\sin \left(\left(\omega_{i}-\omega_{0}\right) t-\hat{\varphi}(t)\right)+\sin \left(\left(\omega_{i}+\omega_{0}\right) t+\hat{\varphi}(t)\right)\right]
$$

with frequencies

$$
\sum_{i=1}^{N}\left(\omega_{i}-\omega_{0}-\frac{d \hat{\varphi}(t)}{d t}\right)
$$

and

$$
\sum_{i=1}^{N}\left(\omega_{i}+\omega_{0}+\frac{d \hat{\varphi}(t)}{d t}\right)
$$

The output frequencies may be rewritten

$$
\begin{aligned}
\left(\omega_{m}-\omega_{0}-\frac{d \hat{\varphi}(t)}{d t}\right) & +\sum_{i=1}^{m-1}\left(\omega_{i}-\omega_{0}-\frac{d \hat{\varphi}(t)}{d t}\right)+\sum_{i=m+1}^{N}\left(\omega_{i}-\omega_{0}-\frac{d \hat{\varphi}(t)}{d t}\right) \\
& +\sum_{i=1}^{N}\left(\omega_{i}+\omega_{0}+\frac{d \hat{\varphi}(t)}{d t}\right)
\end{aligned}
$$

where

$\omega_{m}-$ the input frequency to be tracked.

$\frac{d \hat{\varphi}(t)}{d t}-$ the derivative of the estimated VCO phase.

The goal is to make the term $\left(\omega_{0}-\frac{d \hat{\varphi}(t)}{d t}\right)$ equal to $\omega_{m}$.

When the phase locked loop works properly, the term $\left(\omega_{0}-\frac{d \hat{\varphi}(t)}{d t}\right)$ becomes very small.

The last term in (35) is the sum of the input and output frequencies and may be removed by inserting the lowpass filter (36) between the phase detector and the loop filter, see Figure 2.

$$
\frac{1}{1+T_{V C O} s}
$$

The loop operating frequency $\omega_{L}$ is defined as the frequency band where the PLL is designed to provide good acquisition. The controller is used to remove the second and third term in (35) but also to achieve

-insensitive to other vibration frequencies

- good acquisition behaviour for the input frequency to be tracked

-insensitive to measurement noise of the input signal 
ESA Contract No. 4834/81, 1990 suggested the controller to be taken as

$$
F(s)=\frac{K\left(1+T_{2} s\right)}{T_{1} s\left(1+T_{3} s\right)}
$$

where

$$
T_{3}=\frac{\sec \varphi-\tan \varphi}{\omega_{L}}, \quad T_{2}=\frac{1}{\omega_{L}^{2} T_{3}}, \quad T_{1}=\frac{1}{\omega_{L}^{2}(\sec \varphi-\tan \varphi)}
$$

where $\varphi$ is the phase margin of the operating frequency (normally set to 45 degrees) and $K$ is the loop gain. Choosing the above time constants can be shown to give an acquisition range $\approx 1.5 \omega_{L}$ and an acquisition time $\approx \frac{4 \pi}{\omega_{L}}$.

\section{Simulation results}

The space structure described in Section 2 has been modelled in MATLAB and simulated. The space structure consists of a flexible beam connected to a rigid body. The flexible beam is modelled as a 4-modes Euler-Bernoulli beam, the vibration frequencies are given in Table 1. The process is limited to contain measurement noise only, and the measurement noise is assumed to be white, i.e. Gaussian distributed white noise.

\section{Excitation}

Disturbances or attitude corrections will force the spacecraft to vibrate. Different types of excitation may be considered. In this paper excitation will be limited to initial conditions, i.e. the beam is initially bent according to Table 2 and released at time zero. These initial conditions are known to excite all four modes (Skullestad, 1995). Excitation is important since those vibrations not sufficiently excited (persistently excited) may probably never be identified or poorly identified.

\section{Measurements}

The vibration frequencies can be identified based on measurements from different types of sensors. An accelerometer, a strain-gauge and a remote attitude measurement sensor are highly actual sensors. This paper deals with accelerometer measurements only. The accelerometer is located at the end of the beam. Accelerometer output will be corrupted from both bias and noise. The bias forces the mean value to deviate from zero, but it is easy to estimate the mean and then remove it from the measurements. Measurement noise will, however, have significant effect and the accelerometer is modelled as a linear sensor with Gaussian distributed random noise. The measurement noise is given as 1-sigma values. The mathematical model, that generates the measure-

Table 2. Initial displacement of the beam

\begin{tabular}{ll}
\hline Mode & Displacement (m) \\
\hline 1 & $0 \cdot 05$ \\
2 & $0 \cdot 005$ \\
3 & $0 \cdot 005$ \\
4 & $0 \cdot 001$ \\
\hline
\end{tabular}


Mode 1

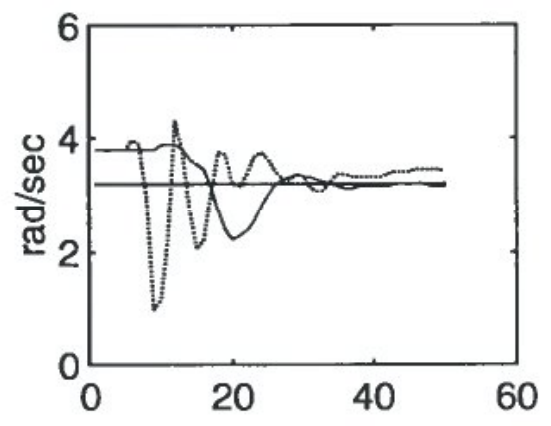

Mode 3

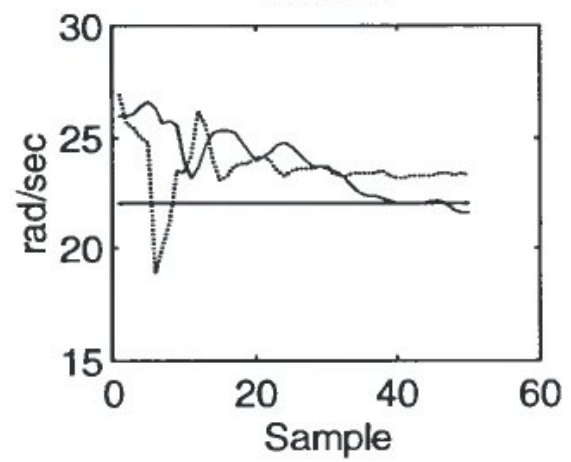

Mode 2
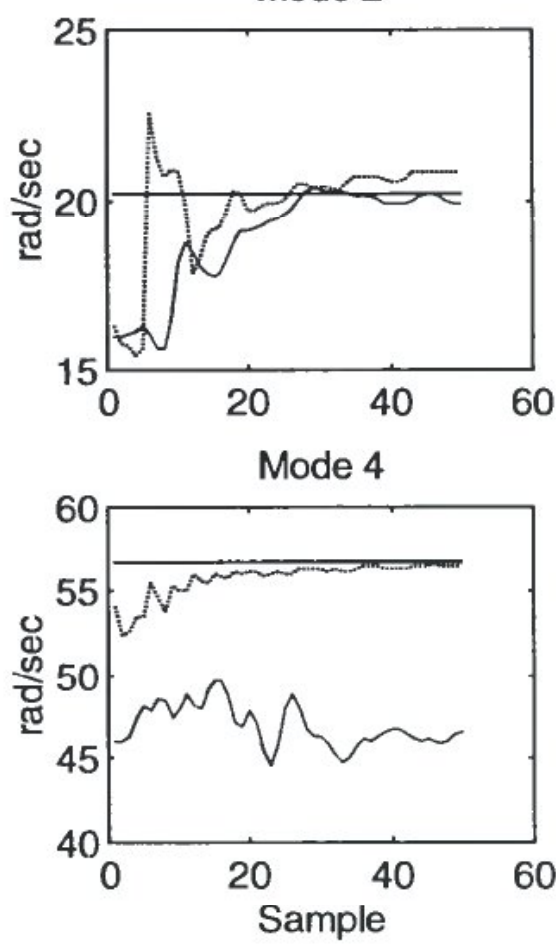

Figure 3a, b,c,d. Augmented Kalman filter (dotted line) and Weighted Kalman filter (solid line).

ments, is continuous, but in the case of the Kalman filters and PEM analysis, the measurements are discretized to match the discrete identification models. The sampling interval is taken as $0 \cdot 02 \mathrm{sec}$. The data record length varies between $20-256$ samples. The measurement noise varies between $0 \cdot 1 \mu \mathrm{g}-0 \cdot 1 \mathrm{~g}$ (1-sigma).

\subsection{Frequency identification using an augmented Kalman filter}

(8) is discretized and used as the Kalman filter model. The measurement noise added to the accelerometer output is $10 \mu \mathrm{g}$. The chosen spacecraft model does not include process noise, and the process noise of the Kalman filter is taken to be small. A Kalman filter may utilize position measurement, velocity measurement, acceleration measurement or a combination of these three. This paper presents results from position measurements, and since the chosen sensor is an accelerometer, the accelerometer signal is integrated twice. Position measurements will often emphasize low frequency vibrations, while acceleration measurements emphasize high frequency vibrations (Skullestad, 1995). Figure 3a,b,c,d, dotted lines, show the estimated frequencies versus time. True frequencies are marked as constant lines. Different process and measurement variances, initial frequency guesses and covariance matrices were tried. Figure 3 is based on the following initial conditions:

Process noise variance, $Q_{d}=0.0001$. Measurement noise variance, $R_{d}=0.01$.

Initial state covariance matrix, $\mathrm{P}=\operatorname{diag}(00000000012040200)$. 
Table 3. True and a priori frequencies

\begin{tabular}{lclll}
\hline True vibration frequencies $(\mathrm{rad} / \mathrm{sec})$ & $3 \cdot 23$ & $20 \cdot 24$ & $22 \cdot 00$ & $56 \cdot 67$ \\
A priori frequencies $(\mathrm{rad} / \mathrm{sec})$ & $3 \cdot 80$ & $16 \cdot 00$ & $26 \cdot 00$ & $46 \cdot 00$ \\
Deviation (\%) & $17 \cdot 6$ & $20 \cdot 9$ & $18 \cdot 2$ & $18 \cdot 8$ \\
\hline
\end{tabular}

It is assumed that the vibration frequencies of the spacecraft are known with an uncertainty of approximately $30 \%$. Table 3 gives the true vibration frequencies and the initial values given as a priori information to the filter, also the initial frequency deviation percentage is shown.

\subsection{Frequency weighting}

The augmented filter, Figure 3, dotted lines, gave relatively inaccurate estimates of modes 1,2 and 3 . It will be interesting to see if a frequency weighted filter is able to improve these estimates. The frequency weighted Kalman filter makes use of the continuous weighting filter, (38). (38) is weighted to emphasize modes 1,2 and 3 .

$$
H(s)=\frac{K^{*} \omega_{0}^{2}}{s^{2}+2 \xi \omega_{0} s+\omega_{0}^{2}}
$$

where $K=10, \omega_{0}=3, \xi=0 \cdot 2$.

The weighting filter, (38), is discretized to accommodate the discrete Kalman filter. The initial conditions are as for the augmented Kalman filter. The additional noise variance, due to the weighting filter, is chosen as $R_{f}=0 \cdot 005$. The additional states in the initial covariance matrix are set to zero. Figure 3a,b, c, d, solid lines, show the estimated vibration frequencies against time for the frequency weighted Kalman filter. The result is as expected, frequency weighting makes the estimates of modes 1,2 and 3 more accurate. The frequency weighted Kalman filter gives, due to the chosen lowpass filter, a poorer mode 4 estimate.

\section{Summary of the augmented and the weighted Kalman filter}

Both the augmented and the frequency weighted Kalman filters are sensitive to measurement noise, and do not work properly for low signal-to-noise ratios. Increasing the measurement noise to $100 \mu \mathrm{g}$ significantly decreases the accuracy of the frequency estimates. Tuning the filter may improve the estimates, but experience shows that proper tuning is very time consuming. It may become difficult to achieve accurate frequency estimates of several vibration modes using one Kalman filter, if:

(a) some of the signals are closely spaced in frequency and/or spread over a wide frequency range;

(b) high and low energy vibrations are mixed together.

An augmented Kalman filter normally pays great attention to high energy vibrations and the low energy vibrations will be difficult to estimate.

Frequency weighting makes it possible to emphasize user specified frequency regions and frequencies of low energy signals may be more easily estimated.

\subsection{Frequency identification using a Gauss-Newton numerical search}

This chapter shows identification of vibration frequencies using Gauss-Newton numerical search. The satellite is excited from the initial conditions shown in Table 2. 
Table 4. True and a priori frequencies

\begin{tabular}{lrrrr}
\hline True vibration frequencies $(\mathrm{rad} / \mathrm{sec})$ & $3 \cdot 23$ & $20 \cdot 24$ & $22 \cdot 00$ & $56 \cdot 67$ \\
A priori frequencies (rad/sec) & $2 \cdot 65$ & $16 \cdot 73$ & $17 \cdot 32$ & $52 \cdot 91$ \\
Deviation (\%) & $17 \cdot 60$ & $20 \cdot 90$ & $18 \cdot 20$ & $18 \cdot 80$ \\
\hline
\end{tabular}

Table 5. Identified vibration frequencies using the function, PEM

\begin{tabular}{lllll}
\hline True vibration frequencies $(\mathrm{rad} / \mathrm{sec})$ & $3 \cdot 23$ & $20 \cdot 24$ & $22 \cdot 00$ & $56 \cdot 67$ \\
Estimated frequencies $(\mathrm{rad} / \mathrm{sec})$ & $3 \cdot 20$ & $20 \cdot 25$ & $22 \cdot 00$ & $56 \cdot 61$ \\
\hline
\end{tabular}

Measurements from an accelerometer located at the end of the beam have been added $1000 \mu \mathrm{g}$ measurement noise. (8) represents the Kalman filter model. States 9, 10, 11 and 12, i.e. the parameter models have been removed. The resulting model is then converted to the innovation type Kalman filter structure (31), that can be utilized by the function PEM in the MATLAB System Identification Toolbox.

(31) only requires one noise sequence to describe both the process and measurement noise. The Kalman filter gain can be taken as the steady state gain, which make the computation quicker. The steady state Kalman gain is calculated from the MATLAB function $L Q E$ with process noise $Q_{d}=N(0,0 \cdot 001)$ and measurement noise $R_{d}=N(0 \cdot 4)$. These values are chosen after several experiments, i.e. after tuning the filter. The Gauss-Newton numerical search method is relatively robust with respect to deviations in the Kalman gain. So, 'Tuned filter' may not be the right expression in connection with numerical search methods, since even with the same process statistics, the variance of the measurements used in the Kalman gain calculation can vary as much as 2-20 without significant degeneration of the parameter estimates. Initial estimates are important, when using the function PEM to estimate parameters from a state space model, otherwise the model may be non-observable/non-controllable.

Table 4 shows the initial guesses of each vibration frequency (a priori frequencies), the true frequencies and the frequency deviation in percent.

Table 5 shows the identified frequencies using the function, PEM. The true frequencies are also shown.

\section{Summary}

A Gauss-Newton numerical search method often gives more accurate parameter estimates than augmented and frequency weighted Kalman filters, especially for low and medium high signal-to-noise ratio (SNR) measurements. The method is robust with respect to the chosen process and measurement noise and the Kalman filter gain. Decreasing the measurement noise caused no problems. Increasing the measurement noise up to $10000 \mu \mathrm{g}$, slightly decreases the frequency accuracy, and local minimas more often occur. A problem with numerical search is the demanding calculation.

Accurate initial conditions seemed to contribute little to improve the parameter estimates. However, reasonable accurate initial conditions are necessary to achieve a stable Kalman filter. Local minima are often encountered, especially for low SNR measurements, but may happen even for high SNR measurements. Several experiments with different initial conditions reduce the risk of choosing the wrong parameter values. 
Table 6. PLL parameter settings

\begin{tabular}{|c|c|c|c|c|c|c|c|c|}
\hline \multicolumn{3}{|c|}{ Input parameters } & \multicolumn{6}{|c|}{ Phase locked loop parameters } \\
\hline $\begin{array}{l}\text { Vibration } \\
\text { mode }\end{array}$ & $\begin{array}{c}\text { True } \\
\text { frequency } \\
(\mathrm{rad} / \mathrm{s})\end{array}$ & $\begin{array}{c}\text { A priori } \\
\text { frequency } \\
(\mathrm{rad} / \mathrm{s})\end{array}$ & $\begin{array}{c}\omega_{\mathrm{L}} \\
(\mathrm{rad} / \mathrm{s})\end{array}$ & $\mathrm{T}_{\text {vco }}$ & $T_{1}$ & $\mathrm{~T}_{2}$ & $\mathrm{~T}_{3}$ & K \\
\hline 1 & $3 \cdot 23$ & $2 \cdot 80$ & 0.6 & $0 \cdot 8$ & $5 \cdot 7$ & $3 \cdot 7$ & 0.64 & 1 \\
\hline 2 & $20 \cdot 24$ & 16.00 & $4 \cdot 0$ & 0.12 & 0.15 & 0.6 & $0 \cdot 10$ & 1 \\
\hline 3 & $22 \cdot 00$ & $18 \cdot 00$ & $4 \cdot 0$ & $0 \cdot 12$ & 0.15 & $0 \cdot 6$ & $0 \cdot 10$ & 1 \\
\hline 4 & 56.67 & $52 \cdot 00$ & $11 \cdot 3$ & 0.045 & 0.02 & $0-22$ & 0.04 & 1 \\
\hline
\end{tabular}

\subsection{Phase locked loop}

The space structure includes four vibration modes. Thus, four PLLs are required to identify these vibration frequencies. Each of the four PLLs are first tested separately using single sinusoidals with the true frequencies shown in Table 6. The measurement noise is set to zero.

The input-filter $\mathrm{A}(\mathrm{s})=1$. The time constants in the low-pass filter and the controller are changed according to Table 6, also the a priori chosen PLL frequencies are shown. The frequency band where the PLL provide good acquisition is shown as $\omega_{\mathbf{L}}$. The chosen time constants give an acquisition range of $\approx 2 \omega_{\mathrm{L}}$.

Figure $4 a, b, c, d$ show the estimated frequencies of the four PLL circuits, subjected to single sinusoidals.
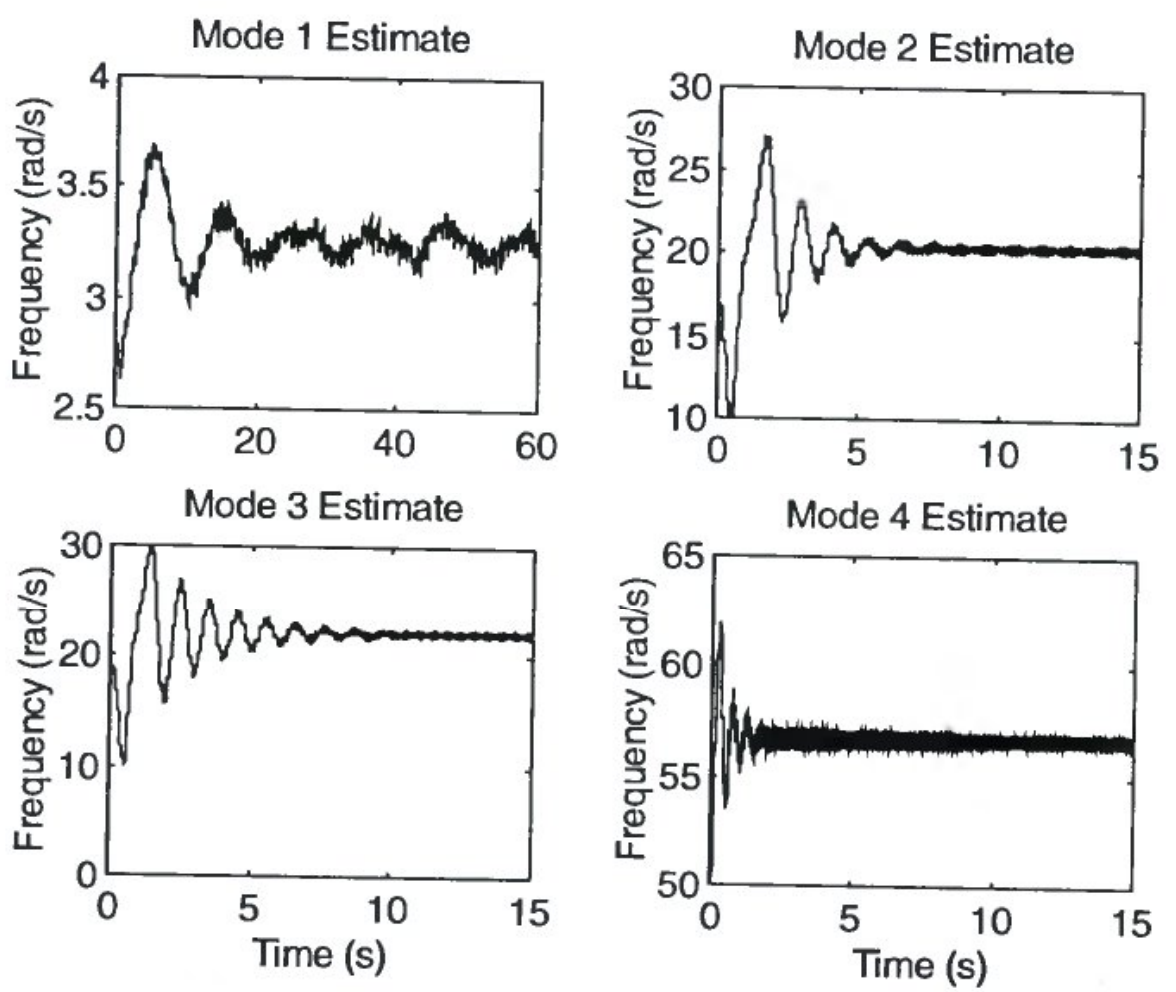

Figure 4a, b, c, d. Separately estimated vibration frequencies using PLLs. 

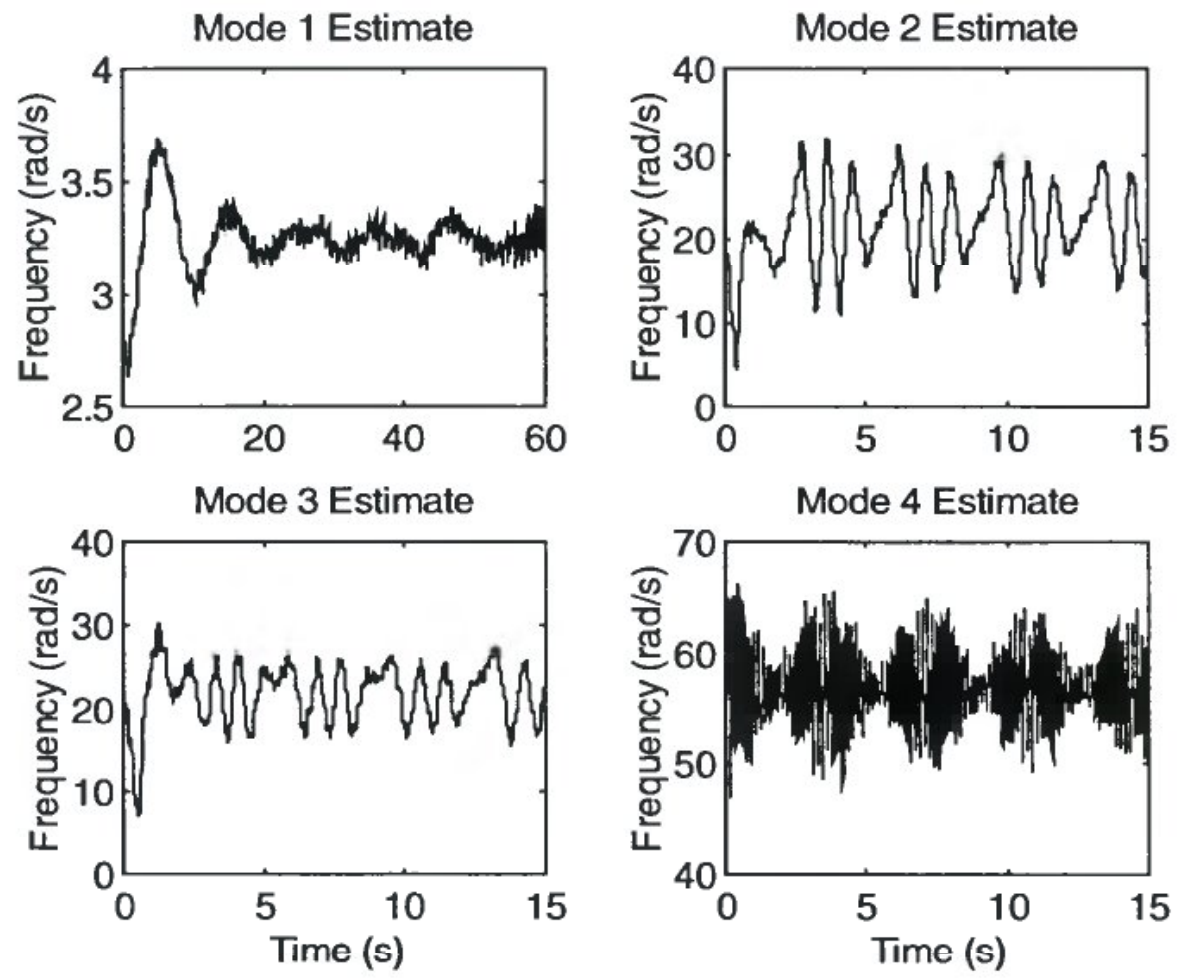

Figure 5a, b, c, d. Vibration frequency estimates using PLLs.

The frequency estimate of mode 1 is slightly noisy. The frequencies of modes 2 and 3 are also noisy, but in average relatively accurately estimated. The same characteristic may be given to the frequency estimate of mode 4. Careful parameter tuning will, as shown later, to some extent improve the frequency estimates. ESA Contract No. 4834/81, 1990 suggest a tuned filter connected to the output of the PLLs. Another approach may simply be to connect a low-pass filter to the output of the PLLs.

Figure 5 shows a more realistic situation, where the accelerometer located at the end of the beam, which measures the true frequencies shown in Table 6, is connected to all four PLLs simultaneously. The initial frequency guesses and the other PLL parameters are according to Table 6 . The measurement noise is set to zero.

Figure $5 \mathrm{a}, \mathrm{b}, \mathrm{c}, \mathrm{d}$ show that the frequency estimates become less accurate when the input signal contain interfering frequencies.

A Chebychef filter is added to each PPL and act as the input-filters. The inputfilters that are connected to the different PLLs are chosen as Chebychef band-pass filters of order 3, with centre frequencies at $2.80(\mathrm{rad} / \mathrm{s}), 16.00(\mathrm{rad} / \mathrm{s}), 18.00(\mathrm{rad} / \mathrm{s})$, $52.00(\mathrm{rad} / \mathrm{s})$ and bandwidths $5(\mathrm{rad} / \mathrm{s}), 9(\mathrm{rad} / \mathrm{s}), 9(\mathrm{rad} / \mathrm{s}) 12(\mathrm{rad} / \mathrm{s})$, respectively. Figure 6 shows the resulting frequency estimates.

Figure $6 a, b, c, d$ show the frequency estimates of modes 1 and 4 are improved using input-filters, while the frequency estimates of modes 2 and 3 are only moderately improved. The reason for this is that the PLL circuits dedicated modes 2 and 3 have input-filters that interfere. The initial guess of the frequencies of modes 2 and 3 are $16.00 \mathrm{rad} / \mathrm{s}$ and $18.00 \mathrm{rad} / \mathrm{s}$, respectively, while the true frequencies are $20.24 \mathrm{rad} / \mathrm{s}$ and $22 \mathrm{rad} / \mathrm{s}$, respectively. Those large frequency uncertainties require input-filter 

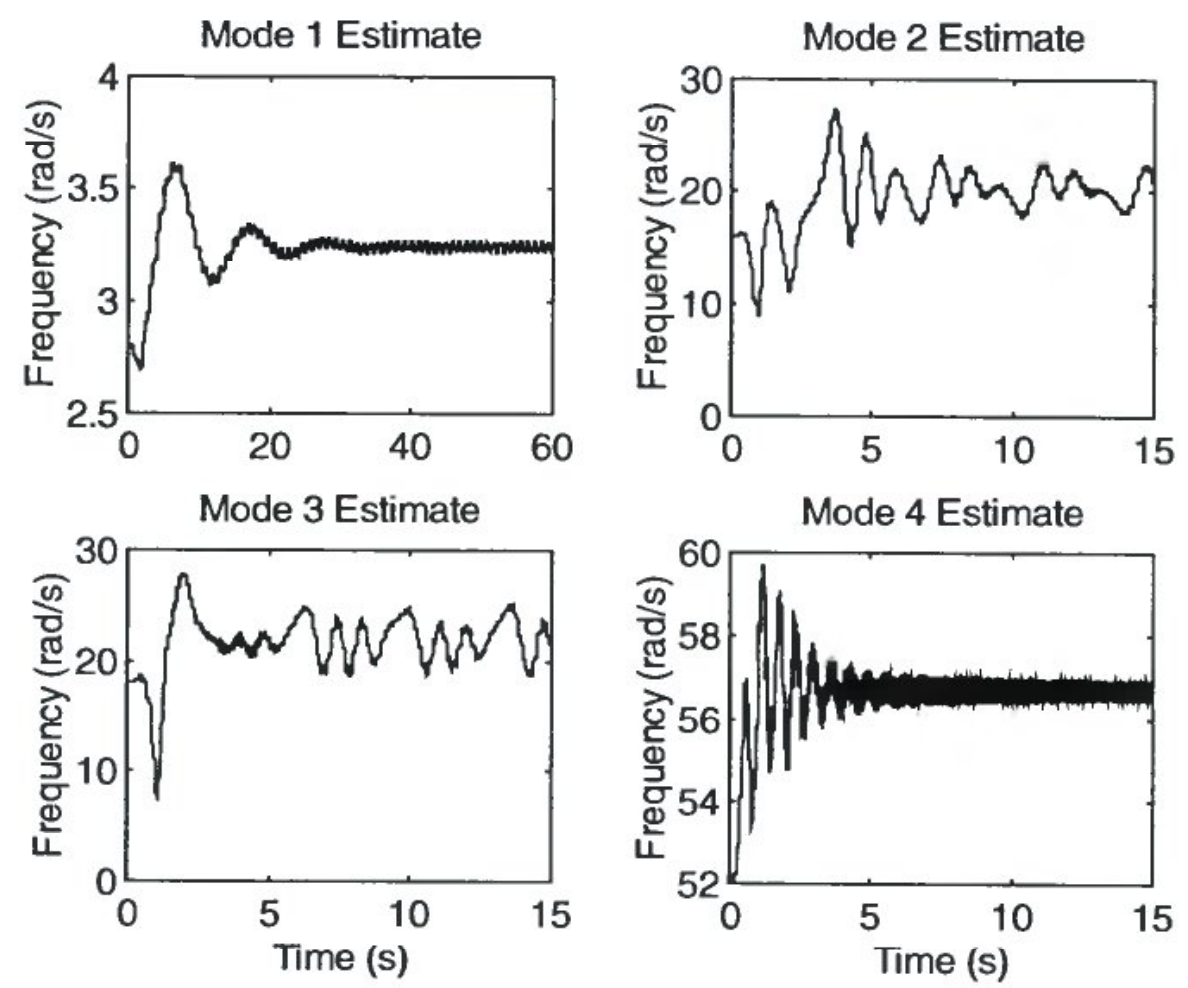

Figure 6a, b, c, d. Vibration frequency estimates using PLLs and input-filters.

bandwidths of at least 7-8 rad/s, which strongly interfere with each other. Less initial frequency uncertainty allow more narrow pass-band filters and, hence, more accurate frequency estimates. The improved frequency accuracy of mode 1 shown in Figure 6a, compared to Figures $4 \mathrm{a}$ and $5 \mathrm{a}$, also comes from better parameter tuning of the mode 1 controller, i.e. $\mathrm{K}$ in Table 6 is increased to 5 .

The input signal of the PLL circuits used to generate Figure 6 is noise free. Accelerometer signals will for sure contain noise and $1000 \mu \mathrm{g}$ measurement noise is added to the accelerometer output. This noise level influences to a small extent the frequency estimates and the measurement noise is increased to $10000 \mu \mathrm{g}$. The obtained frequency estimates, not shown, become close to those documented in Figure 6.

The PLLs including the input-filters manage to suppress the measurement noise and the frequency estimates of modes $1,2,3$ and 4 are still close to the simulations without measurement noise. Increasing the noise to $100000 \mu \mathrm{g}$ worsen the frequency estimates.

\section{Summary}

The PLL gives relatively accurate frequency estimates, but is sensitive to interfering signals that have frequencies within the frequency acquisition range. Input-filtering in order to reduce the effect of interfering signals improves the frequency estimation. Also careful tuning of the controller improves the PLL accuracy. Measurement noise influences the PLL results, but may be reduced using input filtering. 


\section{Conclusion}

Augmented Kalman filters pay great attention to high energy modes and low energy modes may be difficult to identify. Frequency weighted Kalman filters make it possible to emphasize user specified frequency regions and frequencies in low energy signals may be more easily estimated.

Both the augmented and the frequency weighted Kalman filters are sensitive to measurement noise and do not work properly for low and medium high SNR measurements.

PEM using a Gauss-Newton numerical search to perform the parameter identification is more robust to measurement noise. Accurate frequency estimates may be achieved for medium SNR measurements and even for low SNR measurements. Local minima occur especially for low SNR measurements, and several experiments using different initial conditions reduce the risk of choosing the wrong parameters. Numerical search is computationally demanding.

The PLL gives relatively accurate frequency estimates, but is sensitive to interfering signals. Narrow frequencies may be separated using a combination of properly designed controllers and input-filters, but interfering signals often will prevent accurate frequency estimates. The effect of measurement noise is reduced using input-filtering and PLL frequency estimation may be used even for low SNR measurements.

\section{REFERENCES}

Anderson, B. D. O. and Moore, J. B. (1989). Optimal Control. Prentice Hall.

BAR REPORT (1990). Study of Disturbance Torque Cancellation and Control. Volume 2. ESA Contract No. 4834/81.

De Lafontaine, J. (1990). Flexible Mode Dynamic Equations. Personal communications. GelB, A. (1974). Applied Optimal Estimation. MIT Press.

GoluB, G. H. and VAN LOAN, C. S. (1990). Mechanical Vibrations. Addison Wesley.

Skullestad. A. A. (1995). Identification of Vibration Parameters in a Space Structure. PhD Thesis, University of Oslo.

SöDerström, T. and Stolca, P. (1989). System Identification. Prentice Hall.

Williamson, D. (1991). Digital Control and Implementation. Prentice Hall. 